\title{
Correlation between Telomere Length and Chronic Obstructive Pulmonary Disease- Related Phenotypes: Results from the Chronic Obstructive Pulmonary Disease in Dusty Areas (CODA) Cohort
}

\author{
Da Hye Moon, M.D., Ph.D. ${ }^{1}{ }^{\circledR}$, Jeeyoung Kim, Ph.D. ${ }^{2}$, Myoung Nam Lim, Ph.D. ${ }^{3}$, So Hyen Bak, M.D., \\ Ph.D. ${ }^{4}$ and Woo Jin Kim, M.D., Ph.D. ${ }^{1,2}$ (i) \\ ${ }^{1}$ Department of Internal Medicine, Kangwon National University Hospital, Chuncheon, ${ }^{2}$ Department of Internal Medicine and \\ Environmental Health Center, Kangwon National University School of Medicine, Chuncheon, ${ }^{3}$ Biomedical Research Institute, \\ Kangwon National University, Chuncheon, ${ }^{4}$ Department of Radiology, Kangwon National University Hospital, Kangwon \\ National University of School of Medicine, Chuncheon, Republic of Korea
}

Background: Chronic obstructive pulmonary disease (COPD) is a common chronic respiratory disease with increased prevalence in the elderly. Telomeres are repetitive DNA sequences found at the end of the chromosome, which progressively shorten as cells divide. Telomere length is known to be a molecular marker of aging. This study aimed to assess the relationship between telomere length and the risk of COPD, lung function, respiratory symptoms, and emphysema index in Chronic Obstructive Pulmonary Disease in Dusty Areas (CODA) cohort.

Methods: We extracted DNA from the peripheral blood samples of 446 participants, including 285 COPD patients and 161 control participants. We measured absolute telomere length using quantitative real-time polymerase chain reaction. All participants underwent spirometry and quantitative computed tomography scan. Questionnaires assessing respiratory symptoms and the COPD Assessment Test was filled by all the participants.

Results: The mean age of participants at the baseline visit was $72.5 \pm 7.1$ years. Males accounted for $72 \%$ (321 participants) of the all participants. The mean telomere length was lower in the COPD group compared to the non-COPD group (COPD, $16.81 \pm 13.90 \mathrm{~kb}$; non-COPD, $21.97 \pm 14.43 \mathrm{~kb}$ ). In COPD patients, $112(75.7 \%)$ were distributed as tertile 1 (shortest), 91 $(61.1 \%)$ as tertile 2 and $82(55 \%)$ as tertile 3 (longest). We did not find significant associations between telomere length and lung function, exacerbation, airway wall thickness, and emphysema index after adjusting for sex, age, and smoking status.

Conclusion: In this study, the relationship between various COPD phenotypes and telomere length was analyzed, but no significant statistical associations were shown.

Keywords: Telomere Length; Chronic Obstructive Pulmonary Disease; Phenotype

Address for correspondence: Woo Jin Kim, M.D., Ph.D.

Department of Internal Medicine, Kangwon National University School of Medicine, 1 Gangwondaehak-gil, Chuncheon 24341, Republic of Korea

Phone: 82-33-258-9364, Fax: 82-33-255-6567, E-mail: pulmo2@kangwon.ac.kr

Received: Jan. 24, 2021, Revised: Apr. 10, 2021, Accepted: May. 12, 2021, Published online: May. 13, 2021

@(t) is identical to the Creative Commons Attribution Non-Commercial License (http://creativecommons.org/licenses/by-nc/4.0/). 


\section{Introduction}

Chronic obstructive pulmonary disease (COPD) is a major and increasing global health problem with large healthcare costs ${ }^{1}$. COPD is a heterogeneous disease, it comprises of emphysema in the lungs parenchyma, large central airway inflammation, mucociliary dysfunction, bronchiolitis, and small airway structural changes ${ }^{2}$. Airflow limitation, measured by reduced forced expiratory volume in 1 second $\left(\mathrm{FEV}_{1}\right)$, progresses very slowly over several decades. Therefore most patients with symptomatic COPD are in the late middle-aged or elderly. An accelerated rate of lung function decline with age is one of the central pathophysiological characteristics of COPD. Therefore COPD prevalence increases with age ${ }^{1}$.

Telomeres are repetitive DNA sequences, with high G-C content, at the end of chromosome protection. They protect chromosomal ends from being recognized as double-strand breaks and therefore protecting them from end-to-end fusion and degradation ${ }^{3}$. DNA polymerases cannot fully replicate chromosomes, because one RNA primer remains on each daughter DNA strand. This loss of base pairs is a partial consequence of the end-replication problem ${ }^{4}$. Therefore, DNA cannot be duplicated at the end of the chromosome. Each duplication results in a gradual shortening of telomeres, until a critical length is reached at which point cells undergo apopto$\mathrm{sis}^{5}$. In addition, exposure to oxidative stress and inflammation aggravates this shortening ${ }^{6}$. Based on this, some studies have shown that telomere length is a biomarker of cellular aging ${ }^{7,8}$.

Several studies have shown a significant relationship between reduced telomere length in peripheral blood leukocytes and increased risk of malignancy, cardiovascular disease, and diabetes mellitus ${ }^{9-11}$. In these studies, leukocyte telomere length was used as a biomarker of aging based on the hypoth- esis that it reflects the physiological age of individuals. Since COPD is an age-dependent process, assessment of telomere length may be useful for better understanding of the disease pathogenesis.

Since COPD is a heterogeneous disease with variable phenotypes $^{12}$, in the present study, we assumed that the shorter the telomere length, the more depressed the lung function, and the higher the exacerbation rate and degree of emphysema. Accordingly, this study aimed to assess the relationship between telomere length and the lungs function, exacerbation, and visual assessments of emphysema as well as smoking exposure in a Korean COPD cohort residing near cement plants.

\section{Materials and Methods}

\section{Study design and population}

The Participants of the Chronic Obstructive Pulmonary Disease in Dusty Areas (CODA) cohort were analyzed ${ }^{13}$. The CODA study enrolled participants living near six cement plants in the Kangwon and Chungbuk provinces of South Korea. Overall, 504 participants (362 men and 142 women) were enrolled for baseline examinations between 2012 and 2017 (Figure 1). Twenty-five participants whose chest computed tomography (CT) scans showed signs of pneumoconiosis, bronchiectasis or destroyed lung were excluded, four were without completed questionnaire, five without telomere length results, and 23 with extreme telomere length results. Finally, a total of 446 participants were enlisted as eligible participants.

At baseline examinations, a medical interview and survey questionnaire were administered, and spirometry, physical

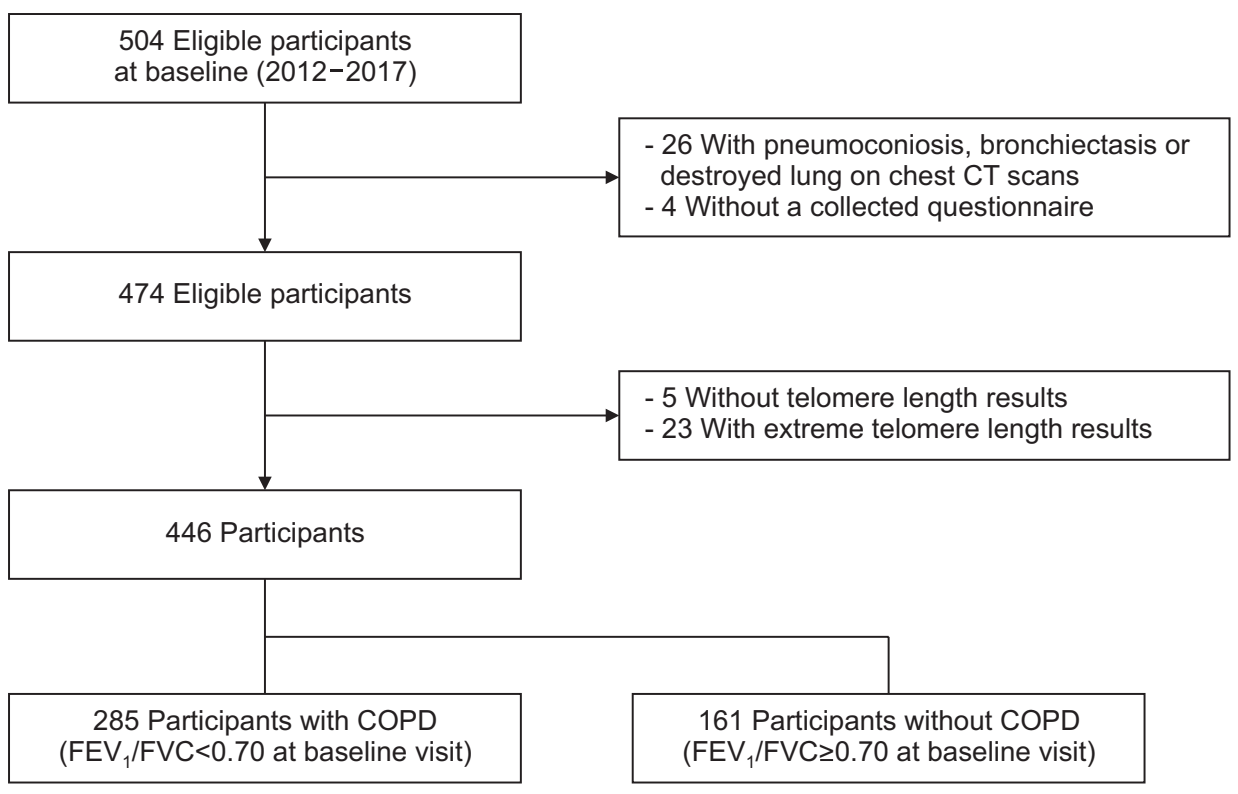

Figure 1. Flowchart of the study population. CT: computed tomography; COPD: chronic obstructive pulmonary disease; $\mathrm{FEV}_{1}$ : forced expiratory volume in $1 \mathrm{sec}-$ ond; FVC: forced vital capacity. 
examination, blood/urine sampling, and CT were performed for all participants. The questionnaire evaluated demographic factors, lifestyle factors, medical history, exacerbation history, and respiratory symptoms during the past year. We defined moderate exacerbation as a history of antibiotics or steroid use for more than two times, and severe exacerbation as more than one hospitalization due to respiratory symptoms within a year.

A written informed consent was given by each participant. This study also received ethical approval from the Institutional Review Board of Kangwon National University Hospital (KNUH 2020-06-007).

\section{Measurement}

Dyspnea was evaluated using the modified Medical Research Council (mMRC) scoring system ${ }^{14}$. Quality of life was assessed using a patients-reported COPD Assessment Test (CAT).

Spirometry was measured yearly using the Easy One Kit (NDD Medizintechnik AG, Zurich, Switzerland), before and after inhalation of $400-\mu \mathrm{g}$ salbutamol. All pulmonary function tests were performed according to the guidelines of the American Thoracic Society/European Respiratory Society ${ }^{15}$.

\section{CT image analysis}

All participants underwent volumetric, thin-section, chest CT at full inspiration and expiration in the supine position. CT images were acquired using a first-generation, dualsource scanner (Somatom Definition, Siemens Healthcare, Forchheim, Germany) in the caudocranial direction using the following parameters: $140 \mathrm{kVp}, 100 \mathrm{~mA}, 0.9-1$ beam pitch, and slice thickness of $0.6 \mathrm{~mm}$ and $3 \mathrm{~mm}$. The CT data were reconstructed using a soft convolution kernel (B30f) ${ }^{16}$. Emphysema was evaluated by automatically extracting all lung images from the chest wall, mediastinum, and large airways. The attenuation coefficients of pixels in these images were then measured. The emphysema index was defined as the volume fraction (\%) of the lung below $-950 \mathrm{HU}$ in full inspira$\operatorname{tion}^{17}$. Mean wall area percentage was calculated as a percentage of the mean values measured in two segmental bronchi (wall area/[wall area+lumen area] $\times 100)^{18}$. The visually defined subtype of COPD were evaluated by two radiologists (with 3 and 11 years of experience) based on the Fleischner Society classification system ${ }^{19}$. Differences in interpretation were resolved through consensus. The subtype were classified into the following seven types: (1) normal, (2) paraseptal emphysema (subjects with substantial paraseptal emphysema), (3) bronchial airway disease, (4) trace centrilobular emphysema, (5) mild centrilobular emphysema, (6) moderate centrilobular emphysema, and (7) confluent and advanced destructive emphysema $^{20}$.

\section{Telomere length measurement}

Venous blood samples were obtained at baseline and DNA was extracted from the buffy coat. Telomere length was measured in DNA isolated from leukocytes. We modified the Cawthon method for relative measurement of telomere length by introducing an oligomer standard to measure absolute telomere length (aTL). In this approach, aTL was calculated by quantitative polymerase chain reaction according to by $\mathrm{O}^{\prime}$ Callaghan and Fenech method ${ }^{21}$, where a standard curve was generated from the fluorescent signals obtained from a series of known concentrations of telomere oligomer DNA $(\mathrm{TTAGG} \times 14)$. The concentration of each test sample was predicted by plotting the fluorescence signal of the sample onto a standard curve. To serve as a reference gene, the concentration of a single copy gene (36B4) DNA in each sample was measured using the same method. Telomere length was calculated as the ratio of telomere DNA length from the standard curve to the 36B4 DNA length.

The telomere lengths of all samples were normalized to a reference cell line control sample, which was evaluated on each plate. Telomere length measurements (kb/genome) were normalized by natural log-transformation. For analysis, we divided the participants into three tertiles according to their telomere lengths.

\section{Statistical analysis}

Four hundred and forty-six participants were divided into were divided into two groups; COPD and non-COPD groups. COPD was defined according to GOLD (Global Initiative for Chronic Obstructive Lung Disease) with post-bronchodilator $\mathrm{FEV}_{1} /$ forced vital capacity $(\mathrm{FVC})<0.70$. For analysis, the telomere length for each group (all participants, COPD group and non-COPD group) were divided into tertile groups. Comparison of baseline characteristics between COPD group and non-COPD group were performed using a Student $t$ test and chi-square test. Categorical variable were described as number (\%). Continuous variables were reported as the mean \pm standard deviation. The lung function, $\mathrm{mMRC}$, and CAT score were evaluated using a general linear model adjusting for age, sex, smoking status, and height. To compare the trend of lung function decline according to the tertile groups, we used a mixed model adjusting for age, sex, and smoking status. Logistic regression was used to find the association between telomere length and COPD exacerbation. Odds ratios (ORs) and 95\% confidence intervals (CI) were calculated using logistic regression model after adjusting for sex, age, and smoking status. For trend tests, individuals were categorized according to telomere length tertile (coded 1-3) with the first tertile consisting of individuals with the shortest telomere lengths. Those with p-values less than 0.05 were considered statistically significant. All analyses were performed using SAS 
version 9.4 (SAS Institute Inc., Cary, NC, USA).

\section{Results}

1. Clinical characteristics, respiratory symptoms and lung functions of the all participants and COPD patients

The clinical characteristics, respiratory symptoms, and lung functions of the 446 participants at baseline are summarized in Table 1. There were 285 participants (63.9\%) in the COPD group and $161(36.1 \%)$ in the non-COPD group. The average telomere length was $18.68 \pm 14.29 \mathrm{~kb}$. The mean telomere length was shorter in the COPD group than in the non-COPD group (COPD, $16.81 \pm 13.90 \mathrm{~kb}$; non-COPD, $21.97 \pm 14.43 \mathrm{~kb}$ ). The mean age of participants at the baseline was $72.5 \pm 7$.1 years and $72 \%$ (321 participants) of the cohort were men (Table 1).

We divided the participants into three groups depending on the telomere length (tertile $1,<8.54$; tertile 2, 8.54-23.54; tertile 3, >23.54). A total of 285 participants (63.9\%) had a $\mathrm{FEV}_{1} /$ $\mathrm{FVC}<0.70$. In COPD patients, $112(75.7 \%)$ were distributed as tertile $1,91(61.1 \%)$ as tertile 2 , and $82(55 \%)$ as tertile 3 (Table

Table 1. Baseline characteristics of the study participants

\begin{tabular}{|c|c|c|c|c|}
\hline Characteristic & Total & COPD & Non-COPD & p-value \\
\hline Participants & 446 & $285(63.9)$ & $161(36.1)$ & \\
\hline Telomere length, kb & $18.68 \pm 14.29$ & $16.81 \pm 13.90$ & $21.97 \pm 14.43$ & $<0.001$ \\
\hline Sex & & & & $<0.001$ \\
\hline Male & $321(72.0)$ & 227 (79.7) & $94(58.4)$ & \\
\hline Female & $125(28.0)$ & $58(20.3)$ & $67(41.6)$ & \\
\hline Age, yr & $72.54 \pm 7.09$ & $72.86 \pm 7.01$ & $71.96 \pm 7.22$ & 0.199 \\
\hline Smoking & & & & $<0.001$ \\
\hline Current & $95(21.3)$ & $74(26.0)$ & $21(13.0)$ & \\
\hline Former & $185(41.5)$ & $134(47.0)$ & $51(31.7)$ & \\
\hline None & $166(37.2)$ & $77(27.0)$ & $89(55.3)$ & \\
\hline Pack-year & $17.40 \pm 23.36$ & $20.22 \pm 25.08$ & $12.26 \pm 18.98$ & 0.001 \\
\hline CAT & $16.20 \pm 9.63$ & $17.11 \pm 9.60$ & $14.60 \pm 9.50$ & 0.008 \\
\hline mMRC & $1.37 \pm 1.14$ & $1.47 \pm 1.14$ & $1.18 \pm 1.10$ & 0.009 \\
\hline \multicolumn{5}{|l|}{ Pre-bronchodilator } \\
\hline FVC, L & $2.86 \pm 0.80$ & $2.89 \pm 0.82$ & $2.79 \pm 0.75$ & 0.195 \\
\hline FVC, \% predicted & $93.46 \pm 19.99$ & $92.28 \pm 20.86$ & $95.57 \pm 18.21$ & 0.095 \\
\hline $\mathrm{FEV}_{1}, \mathrm{~L}$ & $1.86 \pm 0.59$ & $1.74 \pm 0.58$ & $2.08 \pm 0.54$ & $<0.001$ \\
\hline $\mathrm{FEV}_{1}, \%$ predicted & $83.88 \pm 23.29$ & $76.19 \pm 21.07$ & $97.48 \pm 20.73$ & $<0.001$ \\
\hline $\mathrm{FEV}_{1} / \mathrm{FVC}$ & $65.13 \pm 11.49$ & $59.78 \pm 9.22$ & $74.58 \pm 8.68$ & $<0.001$ \\
\hline \multicolumn{5}{|l|}{ Post-bronchodilator } \\
\hline FVC, L & $2.99 \pm 0.80$ & $3.10 \pm 0.81$ & $2.81 \pm 0.75$ & 0.001 \\
\hline FVC, \% predicted & $97.74 \pm 19.21$ & $98.55 \pm 19.36$ & $96.32 \pm 18.92$ & 0.239 \\
\hline $\mathrm{FEV}_{1}, \mathrm{~L}$ & $1.94 \pm 0.59$ & $1.83 \pm 0.57$ & $2.14 \pm 0.56$ & $<0.001$ \\
\hline $\mathrm{FEV}_{1}, \%$ predicted & $87.47 \pm 22.63$ & $80.10 \pm 19.99$ & $100.50 \pm 21.15$ & $<0.001$ \\
\hline $\mathrm{FEV}_{1} / \mathrm{FVC}$ & $65.25 \pm 11.44$ & $58.84 \pm 8.61$ & $76.58 \pm 5.45$ & $<0.001$ \\
\hline IL-8 (n=359) & $18.06 \pm 22.31$ & $16.89 \pm 18.10$ & $21.27 \pm 30.96$ & 0.194 \\
\hline IL-6 (n=359) & $2.52 \pm 3.48$ & $2.51 \pm 3.66$ & $2.57 \pm 2.94$ & 0.867 \\
\hline CRP (n=359) & $0.28 \pm 0.62$ & $0.26 \pm 0.59$ & $0.31 \pm 0.70$ & 0.475 \\
\hline
\end{tabular}

Values are presented as number (\%) or mean \pm SD and analyzed with the $t$ test or chi-square test.

COPD: chronic obstructive pulmonary disease; CAT: COPD Assessment Test; mMRC: modified Medical Research Council; FVC: forced vital capacity; $\mathrm{FEV}_{1}$ : forced expiratory volume in 1 second; IL-8: interleukin-8; IL-6: interleukin-6; CRP: C-reactive protein. 
Table 2. Clinical demographic characteristics, respiratory symptom and lung function of all participants ( $\mathrm{n}=446$ )

\begin{tabular}{|c|c|c|c|c|c|c|}
\hline & Total & $\begin{array}{l}\text { Tertile } 1 \\
(<8.54)\end{array}$ & $\begin{array}{c}\text { Tertile } 2 \\
(8.54-23.54)\end{array}$ & $\begin{array}{l}\text { Tertile } 3 \\
(>23.54)\end{array}$ & $\begin{array}{l}\text { Unadjusted } \\
\text { p trend }\end{array}$ & $\begin{array}{c}\text { Adjusted } \\
\text { p trend }\end{array}$ \\
\hline Participants & 446 & 148 (33.2) & $149(33.4)$ & $149(33.4)$ & & \\
\hline Telomere length, kb & $18.68 \pm 14.29$ & $4.73 \pm 2.52$ & $15.46 \pm 4.53$ & $35.75 \pm 9.43$ & $<0.001$ & \\
\hline COPD & & & & & $<0.001$ & \\
\hline Yes & $285(63.9)$ & $112(75.7)$ & $91(61.1)$ & $82(55.0)$ & & \\
\hline No & $161(36.1)$ & $36(24.3)$ & $58(38.9)$ & $67(45.0)$ & & \\
\hline Sex & & & & & 0.437 & \\
\hline Male & $321(72.0)$ & $112(75.7)$ & $106(71.1)$ & $103(69.1)$ & & \\
\hline Female & $125(28.0)$ & $36(24.3)$ & $43(28.9)$ & $46(30.9)$ & & \\
\hline Age, yr & $72.54 \pm 7.09$ & $72.96 \pm 6.86$ & $71.85 \pm 7.39$ & $72.80 \pm 7.02$ & 0.346 & \\
\hline Smoking & & & & & 0.150 & \\
\hline Current & $95(21.3)$ & $41(27.7)$ & $26(17.5)$ & $28(18.8)$ & & \\
\hline Former & $185(41.5)$ & $61(41.2)$ & $64(42.9)$ & $60(40.3)$ & & \\
\hline None & $166(37.2)$ & $46(31.1)$ & $59(39.6)$ & $61(40.9)$ & & \\
\hline Pack-year & $17.40 \pm 23.36$ & $21.28 \pm 25.45$ & $14.05 \pm 18.87$ & $16.97 \pm 24.82$ & 0.029 & \\
\hline CAT & $16.20 \pm 9.63$ & $16.74 \pm 0.91$ & $16.17 \pm 0.93$ & $15.79 \pm 0.93$ & 0.772 & 0.697 \\
\hline mMRC & $1.37 \pm 1.14$ & $1.31 \pm 0.11$ & $1.38 \pm 0.11$ & $1.33 \pm 0.11$ & 0.863 & 0.855 \\
\hline \multicolumn{7}{|l|}{ Pre-bronchodilator } \\
\hline FVC, L & $2.86 \pm 0.80$ & $2.73 \pm 0.06$ & $2.77 \pm 0.07$ & $2.83 \pm 0.06$ & 0.943 & 0.369 \\
\hline FVC, \% predicted* & $93.46 \pm 19.99$ & $94.16 \pm 1.79$ & $93.53 \pm 1.84$ & $97.07 \pm 1.82$ & 0.176 & 0.249 \\
\hline $\mathrm{FEV}_{1}, \mathrm{~L}$ & $1.86 \pm 0.59$ & $1.76 \pm 0.05$ & $1.82 \pm 0.05$ & $1.88 \pm 0.05$ & 0.410 & 0.143 \\
\hline $\mathrm{FEV}_{1}, \%$ predicted $^{*}$ & $83.88 \pm 23.29$ & $83.50 \pm 2.02$ & $83.78 \pm 2.08$ & $88.66 \pm 2.05$ & 0.034 & 0.077 \\
\hline $\mathrm{FEV}_{1} / \mathrm{FVC}$ & $65.13 \pm 11.49$ & $64.19 \pm 1.00$ & $66.03 \pm 1.03$ & $66.90 \pm 1.02$ & 0.031 & 0.096 \\
\hline \multicolumn{7}{|l|}{ Post-bronchodilator } \\
\hline FVC, L & $2.99 \pm 0.80$ & $2.89 \pm 0.06$ & $2.87 \pm 0.06$ & $2.97 \pm 0.06$ & 0.793 & 0.284 \\
\hline FVC, \% predicted* & $97.74 \pm 19.21$ & $99.38 \pm 1.73$ & $96.80 \pm 1.78$ & $101.64 \pm 1.76$ & 0.081 & 0.086 \\
\hline $\mathrm{FEV}_{1}, \mathrm{~L}$ & $1.94 \pm 0.59$ & $1.84 \pm 0.05$ & $1.89 \pm 0.05$ & $1.96 \pm 0.05$ & 0.492 & 0.135 \\
\hline $\mathrm{FEV}_{1}, \%$ predicted ${ }^{*}$ & $87.47 \pm 22.63$ & $87.23 \pm 1.98$ & $86.68 \pm 2.03$ & $92.20 \pm 2.01$ & 0.027 & 0.052 \\
\hline $\mathrm{FEV}_{1} / \mathrm{FVC}$ & $65.25 \pm 11.44$ & $63.84 \pm 1.01$ & $65.94 \pm 1.03$ & $66.66 \pm 1.02$ & 0.213 & 0.072 \\
\hline IL-8 (n=263) & $16.89 \pm 18.10$ & $16.82 \pm 17.86$ & $15.73 \pm 13.28$ & $18.37 \pm 22.92$ & 0.664 & \\
\hline IL-6 (n=263) & $2.51 \pm 3.66$ & $2.56 \pm 4.18$ & $2.71 \pm 3.49$ & $2.19 \pm 2.95$ & 0.668 & \\
\hline $\mathrm{CRP}(\mathrm{n}=263)$ & $0.26 \pm 0.59$ & $0.27 \pm 0.58$ & $0.29 \pm 0.73$ & $0.22 \pm 0.41$ & 0.740 & \\
\hline \multicolumn{7}{|l|}{ Exacerbation } \\
\hline Moderate & $10(3.5)$ & $3(2.7)$ & $3(3.3)$ & $4(4.9)$ & 0.707 & \\
\hline Severe & $13(4.6)$ & $4(3.6)$ & $4(4.4)$ & $5(6.1)$ & 0.704 & \\
\hline Moderate or severe & $18(6.3)$ & $6(5.4)$ & $6(6.6)$ & $6(7.3)$ & 0.850 & \\
\hline
\end{tabular}

Values are presented as number $(\%)$ or mean \pm SD and analyzed with the $t$ test or chi-square test.

Study participants were divided into three groups based on telomere length, with the first tertile being the shortest and the third tertile being the longest.

Adjusted variables: sex, age, smoking status, height.

*Adjusted variables: sex, age, smoking status.

COPD: chronic obstructive pulmonary disease; CAT: COPD Assessment Test; mMRC: modified Medical Research Council; FVC: forced vital capacity; $\mathrm{FEV}_{1}$ : forced expiratory volume in 1 second; IL-8: interleukin-8; IL-6: interleukin-6; CRP: C-reactive protein. 
2). There were no statistically significant associations between lung function and telomere length after adjusting for sex, age, and smoking status. However, shorter telomere length showed the tendencies that decreased FVC at pre-bronchodilator and FVC, FVC \% predicted at post-bronchodilator in COPD patients ( $\mathrm{p}$ trend, $0.217,0.157$, and 0.319 , respectively) (Table

Table 3. Clinical demographic characteristics, respiratory symptom and pulmonary function of COPD patients $(\mathbf{n}=\mathbf{2 8 5})$

\begin{tabular}{|c|c|c|c|c|c|c|}
\hline & & $\begin{array}{c}\text { Tertile } 1^{*} \\
(<7.39)\end{array}$ & $\begin{array}{c}\text { Tertile 2* }^{*} \\
(7.39-20.58)\end{array}$ & $\begin{array}{l}\text { Tertile } 3^{*} \\
(>20.58)\end{array}$ & $\begin{array}{l}\text { Unadjusted } \\
\text { p trend }\end{array}$ & $\begin{array}{l}\text { Adjusted } \\
\text { p trend }\end{array}$ \\
\hline Participants & 285 & $95(33.3)$ & $95(33.3)$ & $95(33.3)$ & & \\
\hline Telomere length & $18.68 \pm 14.29$ & $4.11 \pm 2.30$ & $12.89 \pm 4.13$ & $33.44 \pm 10.17$ & $<0.001$ & \\
\hline Sex & & & & & 0.206 & \\
\hline Male & $227(79.6)$ & $79(83.2)$ & $70(73.7)$ & $78(82.1)$ & & \\
\hline Female & $58(20.4)$ & $16(16.8)$ & $25(26.3)$ & $17(17.9)$ & & \\
\hline Age & $72.86 \pm 7.01$ & $73.29 \pm 7.04$ & $72.69 \pm 7.32$ & $72.61 \pm 6.73$ & 0.768 & \\
\hline Smoking & & & & & 0.215 & \\
\hline Current & $74(26.0)$ & $28(29.5)$ & $25(26.3)$ & $21(22.1)$ & & \\
\hline Former & $134(47.0)$ & $48(50.5)$ & $38(40.0)$ & $48(50.5)$ & & \\
\hline None & $77(27.0)$ & $19(20.0)$ & $32(33.7)$ & $26(27.4)$ & & \\
\hline Pack-year & $20.30 \pm 25.08$ & $25.31 \pm 27.03$ & $15.05 \pm 19.10$ & $20.71 \pm 27.44$ & 0.020 & \\
\hline CAT & $17.11 \pm 9.60$ & $16.87 \pm 1.18$ & $17.57 \pm 1.21$ & $16.67 \pm 1.27$ & 0.680 & 0.886 \\
\hline mMRC & $1.47 \pm 1.14$ & $1.36 \pm 0.14$ & $1.56 \pm 0.14$ & $1.43 \pm 0.15$ & 0.283 & 0.697 \\
\hline \multicolumn{7}{|l|}{ Pre-bronchodilator } \\
\hline FVC, L & $2.89 \pm 0.82$ & $2.77 \pm 0.08$ & $2.88 \pm 0.09$ & $2.89 \pm 0.09$ & 0.823 & 0.217 \\
\hline $\mathrm{FVC} \%$ predicted $^{\dagger}$ & $92.28 \pm 20.86$ & $93.86 \pm 2.44$ & $96.56 \pm 2.41$ & $96.37 \pm 2.53$ & 0.525 & 0.403 \\
\hline $\mathrm{FEV}_{1}, \mathrm{~L}$ & $1.74 \pm 0.58$ & $1.68 \pm 0.06$ & $1.74 \pm 0.06$ & $1.72 \pm 0.07$ & 0.962 & 0.593 \\
\hline $\mathrm{FEV}_{1}, \%$ predicted $^{\dagger}$ & $76.19 \pm 21.07$ & $77.55 \pm 2.47$ & $79.66 \pm 2.44$ & $77.90 \pm 2.56$ & 0.637 & 0.908 \\
\hline $\mathrm{FEV}_{1} / \mathrm{FVC}$ & $59.79 \pm 9.22$ & $60.31 \pm 1.09$ & $60.10 \pm 1.08$ & $59.51 \pm 1.13$ & 0.853 & 0.552 \\
\hline \multicolumn{7}{|l|}{ Post-bronchodilator } \\
\hline FVC, L & $3.10 \pm 0.81$ & $2.93 \pm 0.08$ & $3.00 \pm 0.08$ & $3.06 \pm 0.08$ & 0.517 & 0.157 \\
\hline $\mathrm{FVC} \%$ predicted $^{\dagger}$ & $98.55 \pm 19.36$ & $99.22 \pm 2.28$ & $100.67 \pm 2.25$ & $102.23 \pm 2.37$ & 0.562 & 0.319 \\
\hline $\mathrm{FEV}_{1}, \mathrm{~L}$ & $1.83 \pm 0.57$ & $1.76 \pm 0.06$ & $1.79 \pm 0.06$ & $1.81 \pm 0.06$ & 0.734 & 0.512 \\
\hline $\mathrm{FEV}_{1}, \%$ predicted $^{\dagger}$ & $80.10 \pm 19.99$ & $81.25 \pm 2.35$ & $82.12 \pm 2.32$ & $81.66 \pm 2.45$ & 0.881 & 0.889 \\
\hline $\mathrm{FEV}_{1} / \mathrm{FVC}$ & $58.84 \pm 8.61$ & $59.46 \pm 1.02$ & $59.33 \pm 1.01$ & $58.97 \pm 1.06$ & 0.931 & 0.695 \\
\hline IL-8 (n=263) & $16.89 \pm 18.10$ & $17.10 \pm 19.12$ & $16.35 \pm 12.85$ & $17.24 \pm 21.65$ & 0.941 & \\
\hline IL-6 (n=263) & $2.51 \pm 3.66$ & $2.51 \pm 4.27$ & $2.91 \pm 3.67$ & $2.06 \pm 2.78$ & 0.311 & \\
\hline CRP (n=263) & $0.26 \pm 0.59$ & $0.25 \pm 0.54$ & $0.33 \pm 0.77$ & $0.20 \pm 0.38$ & 0.378 & \\
\hline \multicolumn{7}{|l|}{ Exacerbation } \\
\hline Moderate & $10(3.5)$ & $3(3.2)$ & $3(3.2)$ & $4(4.2)$ & 0.902 & \\
\hline Severe & $13(4.6)$ & $4(4.2)$ & $4(4.2)$ & $5(5.3)$ & 0.923 & \\
\hline Moderate or severe & $18(6.3)$ & $6(6.3)$ & $6(6.3)$ & $6(6.3)$ & - & \\
\hline
\end{tabular}

Values are presented as number $(\%)$ or mean \pm SD and analyzed with the $t$ test or chi-square test.

Adjusted variables: sex, age, smoking status, height.

*COPD patients were divided into three groups based on telomere length, with the first tertile being the shortest and the third tertile being the longest. ${ }^{\dagger}$ Adjusted variables: sex, age, smoking status.

COPD: chronic obstructive pulmonary disease; CAT: COPD Assessment Test; mMRC: modified Medical Research Council; FVC: forced vital capacity; $\mathrm{FEV}_{1}$ : forced expiratory volume in 1 second; IL-8: interleukin-8; IL-6: interleukin-6; CRP: C-reactive protein. 
3). In the non-COPD group, shorter telomere length in tertile showed tendencies of decreased $\mathrm{FEV}_{1} / \mathrm{FVC}$ at pre-bronchodilator and post-bronchodilator ( $\mathrm{p}$ trend, 0.03 and 0.005 , respectively) (Supplementary Table S1).

2. The relationship between telomere length and lung function decline

In all participants and COPD patients, shorter telomere length in tertile was associated with decreased FVC L at prebronchodilator ( $p$ trend, $<0.001$ and $<0.001$, respectively) (Tables 4,5 ). In non-COPD group, longer telomere length in tertile showed tendencies of increased FVC L at post-bronchodilator ( $p$ trend, <0.001) (Supplementary Table S2).

3. The relationship between telomere length and visual and quantitative CT imaging features

Of the 446 participants, 25 were excluded from the visual assessment of the CT scans due to severe lung distortion and other lung morbidity. Finally, we analyzed 421 participants. Among the 285 patients with COPD, 13 were excluded from the visual assessment of CT scans; only 272 patients were included in the analysis. There were no statistically significant associations between telomere length and emphysema index, mean wall area, and CT subtypes in all participants and COPD patients (Table 6, Supplementary Table S3). In non-COPD participants, decreasing telomere length was associated with increased mean wall area ( $\mathrm{p}$ trend, 0.415) (Supplementary Table S4).

\section{The relationship between telomere length and acute exacerbation}

We did not establish a significant association between telomere length and COPD exacerbation in COPD patients. In multivariable analyses, OR for the shortest versus the longest telomere tertile was 0.707 (95\% CI, 0.153-3.274) for moderate exacerbation and $0.788(0.203-3.057)$ for severe exacerbation (Table 7).

\section{Discussion}

We investigated the relationship between telomere length and the lung function, respiratory symptoms, emphysema

Table 4. Change in FVC and $\mathrm{FEV}_{1}$ according to telomere length (all participants, $\mathrm{n}=446$ )

\begin{tabular}{|c|c|c|c|c|}
\hline & Tertile $1(<8.54)$ & Tertile 2 (8.54-23.54) & Tertile 3 (>23.54) & p trend \\
\hline \multicolumn{5}{|c|}{ Pre-bronchodilator } \\
\hline $\mathrm{FVC}, \mathrm{mL} / \mathrm{yr}$ & $-4.294 \pm 1.707$ & $-5.086 \pm 1.898$ & $-6.044 \pm 2.187$ & $<0.001$ \\
\hline $\mathrm{FEV}_{1}, \mathrm{~mL} / \mathrm{yr}$ & $-2.675 \pm 1.692$ & $-3.796 \pm 1.820$ & $-3.304 \pm 2.000$ & 0.736 \\
\hline \multicolumn{5}{|c|}{ Post-bronchodilator } \\
\hline $\mathrm{FVC}, \mathrm{mL} / \mathrm{yr}$ & $-2.467 \pm 1.577$ & $-3.480 \pm 1.737$ & $-2.578 \pm 1.990$ & 0.952 \\
\hline $\mathrm{FEV}_{1}, \mathrm{~mL} / \mathrm{yr}$ & $-2.199 \pm 0.903$ & $-2.709 \pm 0.975$ & $-1.364 \pm 1.094$ & 0.409 \\
\hline
\end{tabular}

Study participants were divided into three groups based on telomere length, with the first tertile being the shortest and the third tertile being the longest.

Adjusted variables: sex, age, smoking status, height.

FVC: forced vital capacity; $\mathrm{FEV}_{1}$ : forced expiratory volume in 1 second.

Table 5. Change in FVC and $\mathrm{FEV}_{1}$ according to telomere length (COPD patients, $\mathbf{n}=\mathbf{2 8 5}$ )

\begin{tabular}{|c|c|c|c|c|}
\hline & Tertile $1^{*}(<7.39)$ & Tertile $2^{*}(7.39-20.58)$ & Tertile $3^{*}(>20.58)$ & $p$ trend \\
\hline \multicolumn{5}{|c|}{ Pre-bronchodilator } \\
\hline $\mathrm{FVC}, \mathrm{mL} / \mathrm{yr}$ & $-5.652 \pm 2.004$ & $-6.358 \pm 2.248$ & $-7.346 \pm 2.706$ & $<0.001$ \\
\hline $\mathrm{FEV}_{1}, \mathrm{~mL} / \mathrm{yr}$ & $-3.297 \pm 2.005$ & $-5.225 \pm 2.197$ & $-4.206 \pm 2.477$ & 0.679 \\
\hline \multicolumn{5}{|c|}{ Post-bronchodilator } \\
\hline $\mathrm{FVC}, \mathrm{mL} / \mathrm{yr}$ & $-5.003 \pm 1.900$ & $-5.368 \pm 2.114$ & $-4.606 \pm 2.518$ & 0.856 \\
\hline $\mathrm{FEV}_{1}, \mathrm{~mL} / \mathrm{yr}$ & $-2.463 \pm 0.975$ & $-2.958 \pm 1.078$ & $-1.998 \pm 1.269$ & 0.674 \\
\hline
\end{tabular}

Adjusted variables: sex, age, smoking status, height.

*COPD patients were divided into three groups based on telomere length, with the first tertile being the shortest and the third tertile being the longest.

FVC: forced vital capacity; $\mathrm{FEV}_{1}$ : forced expiratory volume in 1 second; COPD: chronic obstructive pulmonary disease. 


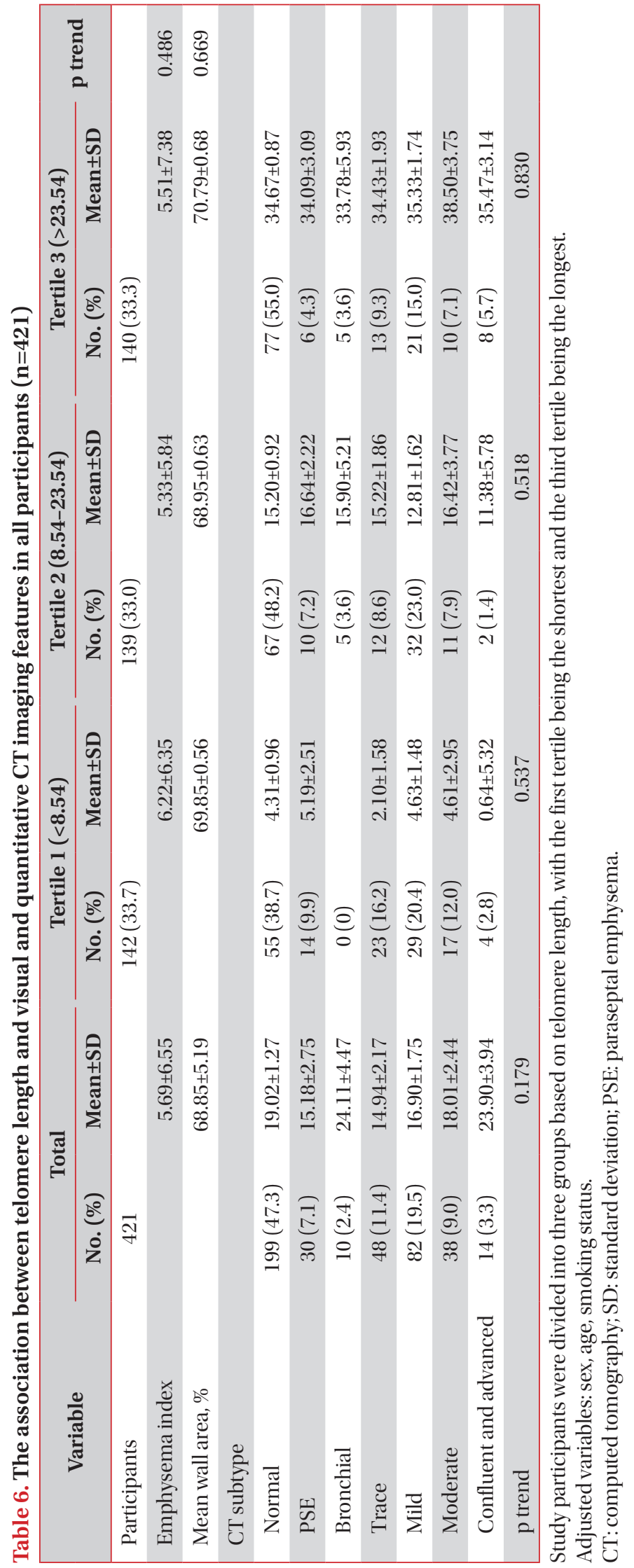


Table 7. The OR of exacerbation according to telomere length (COPD patients, $\mathbf{n}=\mathbf{2 8 5})$

\begin{tabular}{|lccc|}
\hline & Moderate & Severe & Moderate or severe \\
\hline Telomere length & & & \\
\hline Tertile $1^{*}$ vs. tertile $3^{*}$ & $0.707(0.153-3.274)$ & $0.788(0.203-3.057)$ & $0.997(0.307-3.241)$ \\
\hline Tertile 2* vs. tertile $3^{*}$ & $0.681(0.146-3.184)$ & $0.781(0.200-3.050)$ & $0.966(0.295-3.157)$ \\
p-value & 0.859 & 0.919 & 0.998 \\
\hline
\end{tabular}

Adjusted variables: sex, age, smoking status, height.

*COPD patients were divided into three groups based on telomere length, with the first tertile being the shortest and the third tertile being the longest.

OR: odds ratio; COPD: chronic obstructive pulmonary disease.

extent, and airway wall thickness in a Korean COPD cohort living near cement plants. Disappointingly, we did not observe significant associations between telomere length and lung function, exacerbation, airway wall thickness, and emphysema index after adjusting for sex, age, and smoking status. However, although no statistical significances have been shown, some tendencies were shown along the telomere length tertile. It was shown that $\mathrm{FEV}_{1} / \mathrm{FVC}$ (pre-, post-bronchodilator) decreased with shorter telomere length in all participants and in the non-COPD group. The shorter telomere length was shown to decrease FVC (pre-, post-bronchodilator) in COPD group. In all participants and COPD patients, the shorter the telomere length, the lower the FVC (pre-bronchodilator), indicating a decline in the lung function change. On the other hand, in the non-COPD group, the shorter the telomere length, the lower the FVC (post-bronchodilator), indicating an increase in the lung function change. In addition, the shorter the telomere length, the wider the mean wall area in nonCOPD group.

COPD is an age-related disease. An accelerated rate of lung function decline with age is one of the central pathophysiological characteristics of COPD. Previous studies have shown that leukocyte telomeres can be like biomarkers in cellular aging ${ }^{7,22,23}$. Some studies have shown that decreased leukocyte telomere length is associated with a decline in lung function ${ }^{24,25}$. In our study, some tendencies between short telomere length and decreased lung function were shown, but they were not statistically significant.

Rode et al. reported that $\mathrm{FEV}_{1}, \mathrm{FVC}$ and $\mathrm{FEV}_{1} / \mathrm{FVC}$ decreased with decreasing telomere length quartile ( $\mathrm{p}$ trend, $5 \times 10^{-51}, 5 \times 10^{-35}$, and $6 \times 10^{-137}$, respectively) but the associations attenuated after age and multivariable adjustments. In addition, participants with shorter telomeres were 2.06 -fold more likely to develop COPD. The results showed that short telomere length is associated with reduced lung function and an increased prevalence of $\mathrm{COPD}^{6}$. Furthermore, data from seven studies demonstrated highly significant positive association between telomere length and spirometric measurements including $\mathrm{FEV}_{1}\left(\beta=0.0455, \mathrm{p}=1.07 \times 10^{-7}\right.$ with fixed and random effects; $\left.I^{2}=0 \%\right)$, FVC $\left(\beta=0.0401, p=2.07 \times 10^{-7}\right.$ with fixed and random effects; $\left.\mathrm{I}^{2}=0 \%\right)$, and $\mathrm{FEV}_{1} / \mathrm{FVC}(\beta=0.0238$, $\mathrm{p}=5.27 \times 10^{-7}$ with fixed and random effects; $\left.\mathrm{I}^{2}=0 \%\right)^{25}$.

One study reported that shorter leukocyte telomere length may be a biomarker associated with a poor clinical prognosis in COPD, where short telomere length was associated with reduced quality of life and increased risk of exacerbation and mortality in patients with moderate-to-severe $\mathrm{COPD}^{26}$. However, our study did not show any relationship between acute COPD exacerbation and telomere length.

No significant associations were found between the emphysema index, mean wall area and telomere length in the all participants and COPD patients ( $\mathrm{p}$ trend, 0.486, 0.669, 0.553, and 0.328 , respectively). In the sub-analysis, the correlation of the average telomere length and emphysema CT subtype did not show a statistically significant trend in all participants and COPD group ( $\mathrm{p}$ trend, 0.179 and 0.390 , respectively). In addition, we did not observe any association between telomere length and airway wall thickness, emphysema index and visual CT image features in non-COPD group. However, we established that the shorter the telomere length, the wider the mean wall area in non-COPD group.

The MESA (Multi-Ethnic Study of Atherosclerosis) study found that the presence of centrilobular and panlobular emphysema correlated with increased dyspnea and reduced exercise capacity ${ }^{27}$. Airway wall thickness correlated with reduced lung function and increased symptoms in smoker in a cross-sectional study ${ }^{28}$. Image biomarkers have been useful predictors of disease progression. As shown earlier, previous studies showed that telomeres were shorter in peripheral leukocytes of COPD patients and have been related to lung function. However, we did not find other studies on the relationship between CT-based visual assessments and telomere length.

Smoking is a well-known environmental factor that promotes aging and cellular senescence ${ }^{29}$. A large population study showed that a smoking history and the cumulative packyears were associated with telomere length in 46,396 adults ${ }^{30}$. Another study found that smoking promotes shortening of telomere length. Within the same age range, telomere length decreased with age in smokers, irrespective of the presence or 
absence of COPD ( $\mathrm{p}=0.05, \mathrm{r}=-0.27)$. The influence of cumulative smoking exposure on telomere length is further supported by the significant dose-effect relationship demonstrated between pack-years and telomere length $(\mathrm{p}<0.001, \mathrm{r}=-0.45)^{31}$. In this study, when analyzing the relationship between telomere length and pack-year ( $p$ trend, 0.029 and 0.020 , respectively), we observed statistical significances, but we did not establish a trend in all participants and COPD patients.

It is worth noting that this is the first study to examine the relationship between telomere length and the phenotypes of COPD in Koreans. Our study investigated the relationship between telomere length and the extent of emphysema, airway wall thickness, and visual assessment on CT scans. Some studies have demonstrated that emphysema as assessed by CT imaging is a good predictor of mortality in COPD patients at various stages of the disease. Although it is still unclear whether emphysema predisposes COPD patients to such systemic manifestations and whether these systemic manifestations contribute to the development of emphysema, it is apparent that recognizing the extent of emphysema is important in evaluating COPD. Taken together, the evaluation of emphysema seems to be beneficial in the management of COPD ${ }^{32}$.

Nevertheless, this study has several limitations. First, the small sample size resulted in limited power to detect differences between telomere length and COPD-related phenotypes. Further large-scale studies with longer follow-up periods involving several serial assessments are needed to validate our findings.

Second, telomere length is a complex characteristic that is shaped by several factors, including genetic, epigenetic, lifestyle and environmental determinants. The complex interactions of these factors remain unclear ${ }^{33}$. The association between telomere length and environmental, occupational, and medical risk factors has been reported in several crosssectional epidemiological studies ${ }^{34,35}$. However, this study did not consider such environmental and occupational factors. This study consisted participants living in dusty areas near cement plants, thus the results might differ from the general COPD populations. In CODA cohort study, we have records of exposure such as air pollution $\left(\mathrm{PM}_{10}, \mathrm{NO}_{2}\right)$; however, additional analysis could not be carried out due to insufficient number of participants.

Third, we measured the leukocyte telomere length. Regarding this point, it is important to note that a correlation between lung and blood telomere length has not been unequivocally demonstrated. This suggests that the associations between telomere length and various diseases cannot easily be interpreted as causative relationship.

In conclusion, the correlation between lung function, respiratory symptoms, or extent and visual assessment and telomere length were analyzed, we did not find statistically significant results. Further studies are needed on the role of telomere length in COPD pathogenesis, as well as the rela- tionship between telomere length and environmental factors including air pollution.

\section{Authors' Contributions}

Conceptualization: Kim WJ, Moon DH. Methodology: Kim WJ, Moon DH, Kim J. Formal analysis: Lim MN. Data Curation: Lim MN. Software: Lim MN. Validation: Lim MN, Kim WJ, Moon DH. Investigation: Kim WJ, Moon DH, Kim J. Writing - original draft preparation: Moon DH, Kim WJ. Writing - review and editing: Moon DH, Kim J, Lim MN, Bak SH, Kim WJ. Approval of the final manuscript: all authors.

\section{Conflicts of Interest}

No potential conflict of interest relevant to this article was reported.

\section{Funding}

No funding to declare.

\section{Supplementary Material}

Supplementary material can be found in the journal homepage (http://www.e-trd.org).

Supplementary Table S1. Clinical demographic characteristics, respiratory symptom and pulmonary function of nonCOPD patients $(\mathrm{n}=161)$.

Supplementary Table S2. Change in FVC and FEV ${ }_{1}$ according to telomere length (non-COPD patients, $\mathrm{n}=161$ ).

Supplementary Table S3. The association between telomere length and visual and quantitative CT imaging features in COPD patients ( $\mathrm{n}=272)$.

Supplementary Table S4. The association between telomere length and visual and quantitative CT imaging features in nonCOPD participants ( $\mathrm{n}=149)$.

\section{References}

1. Ito $\mathrm{K}$, Barnes PJ. COPD as a disease of accelerated lung aging. Chest 2009;135:173-80.

2. Vestbo J, Anderson W, Coxson HO, Crim C, Dawber F, Edwards L, et al. Evaluation of COPD longitudinally to identify predictive surrogate end-points (ECLIPSE). Eur Respir J 2008; 31:869-73.

3. Blackburn EH. Structure and function of telomeres. Nature 1991;350:569-73 
4. Houben JM, Moonen HJ, van Schooten FJ, Hageman GJ. Telomere length assessment: biomarker of chronic oxidative stress? Free Radic Biol Med 2008;44:235-46.

5. Lee J, Sandford AJ, Connett JE, Yan J, Mui T, Li Y, et al. The relationship between telomere length and mortality in chronic obstructive pulmonary disease (COPD). PLoS One 2012;7: e35567.

6. Rode L, Bojesen SE, Weischer M, Vestbo J, Nordestgaard BG. Short telomere length, lung function and chronic obstructive pulmonary disease in 46,396 individuals. Thorax 2013;68:42935.

7. Mather KA, Jorm AF, Parslow RA, Christensen H. Is telomere length a biomarker of aging? A review. J Gerontol A Biol Sci Med Sci 2011;66:202-13.

8. Armanios M. Telomeres and age-related disease: how telomere biology informs clinical paradigms. J Clin Invest 2013; 123:996-1002.

9. Wu X, Amos CI, Zhu Y, Zhao H, Grossman BH, Shay JW, et al. Telomere dysfunction: a potential cancer predisposition factor. J Natl Cancer Inst 2003;95:1211-8.

10. Calado RT, Young NS. Telomere diseases. N Engl J Med 2009;361:2353-65.

11. Serrano AL, Andres V. Telomeres and cardiovascular disease: does size matter? Circ Res 2004;94:575-84.

12. Silverman EK. Exacerbations in chronic obstructive pulmonary disease: do they contribute to disease progression? Proc Am Thorac Soc 2007;4:586-90.

13. Han Y, Heo Y, Hong Y, Kwon SO, Kim WJ. Correlation between physical activity and lung function in dusty areas: results from the Chronic Obstructive Pulmonary Disease in Dusty Areas (CODA) cohort. Tuberc Respir Dis (Seoul) 2019;82:311-8.

14. Mahler DA, Wells CK. Evaluation of clinical methods for rating dyspnea. Chest 1988;93:580-6.

15. Celli BR, MacNee W; ATS/ERS Task Force. Standards for the diagnosis and treatment of patients with COPD: a summary of the ATS/ERS position paper. Eur Respir J 2004;23:932-46.

16. Bak SH, Kwon SO, Han SS, Kim WJ. Computed tomographyderived area and density of pectoralis muscle associated disease severity and longitudinal changes in chronic obstructive pulmonary disease: a case control study. Respir Res 2019;20:226.

17. Washko GR, Hunninghake GM, Fernandez IE, Nishino M, Okajima Y, Yamashiro T, et al. Lung volumes and emphysema in smokers with interstitial lung abnormalities. N Engl J Med 2011;364:897-906.

18. Kim V, Davey A, Comellas AP, Han MK, Washko G, Martinez $\mathrm{CH}$, et al. Clinical and computed tomographic predictors of chronic bronchitis in COPD: a cross sectional analysis of the COPDGene study. Respir Res 2014;15:52.

19. Lynch DA, Austin JH, Hogg JC, Grenier PA, Kauczor HU, Bankier AA, et al. CT-definable subtypes of chronic obstructive pulmonary disease: a statement of the Fleischner Society. Radiology 2015;277:192-205.
20. Park J, Hobbs BD, Crapo JD, Make BJ, Regan EA, Humphries $\mathrm{S}$, et al. Subtyping COPD by using visual and quantitative CT imaging features. Chest 2020;157:47-60.

21. O'Callaghan NJ, Fenech M. A quantitative PCR method for measuring absolute telomere length. Biol Proced Online 2011;13:3.

22. Levy MZ, Allsopp RC, Futcher AB, Greider CW, Harley CB. Telomere end-replication problem and cell aging. J Mol Biol 1992;225:951-60.

23. Bernadotte A, Mikhelson VM, Spivak IM. Markers of cellular senescence. Telomere shortening as a marker of cellular senescence. Aging (Albany NY) 2016;8:3-11.

24. Clouston SA, Edelman NH, Aviv A, Stewart C, Luft BJ. Shortened leukocyte telomere length is associated with reduced pulmonary function and greater subsequent decline in function in a sample of World Trade Center responders. Sci Rep 2019;9:8148.

25. Albrecht E, Sillanpaa E, Karrasch S, Alves AC, Codd V, Hovatta I, et al. Telomere length in circulating leukocytes is associated with lung function and disease. Eur Respir J 2014;43:983-92.

26. Jin M, Lee EC, Ra SW, Fishbane N, Tam S, Criner GJ, et al. Relationship of absolute telomere length with quality of lfe, exacerbations, and mortality in COPD. Chest 2018;154:26673.

27. Smith BM, Austin JH, Newell JD Jr, D'Souza BM, Rozenshtein A, Hoffman EA, et al. Pulmonary emphysema subtypes on computed tomography: the MESA COPD study. Am J Med 2014;127:94.

28. Dijkstra AE, Postma DS, ten Hacken N, Vonk JM, Oudkerk M, van Ooijen PM, et al. Low-dose CT measurements of airway dimensions and emphysema associated with airflow limitation in heavy smokers: a cross sectional study. Respir Res 2013;14:11.

29. Karrasch S, Holz O, Jorres RA. Aging and induced senescence as factors in the pathogenesis of lung emphysema. Respir Med 2008;102:1215-30.

30. Babizhayev MA, Yegorov YE. Smoking and health: association between telomere length and factors impacting on human disease, quality of life and life span in a large populationbased cohort under the effect of smoking duration. Fundam Clin Pharmacol 2011;25:425-42.

31. Morla M, Busquets X, Pons J, Sauleda J, MacNee W, Agusti AG. Telomere shortening in smokers with and without COPD. Eur Respir J 2006;27:525-8.

32. Halper-Stromberg E, Cho MH, Wilson C, Nevrekar D, Crapo JD, Washko G, et al. Visual assessment of chest computed tomographic images is independently useful for genetic association analysis in studies of chronic obstructive pulmonary disease. Ann Am Thorac Soc 2017;14:33-40.

33. Daniali L, Benetos A, Susser E, Kark JD, Labat C, Kimura M, et al. Telomeres shorten at equivalent rates in somatic tissues of adults. Nat Commun 2013;4:1597.

34. Benetos A, Kark JD, Susser E, Kimura M, Sinnreich R, Chen W, 
et al. Tracking and fixed ranking of leukocyte telomere length across the adult life course. Aging Cell 2013;12:615-21.

35. Starkweather AR, Alhaeeri AA, Montpetit A, Brumelle J, Filler
K, Montpetit M, et al. An integrative review of factors associated with telomere length and implications for biobehavioral research. Nurs Res 2014;63:36-50. 